\title{
Life Historical Perspectives on Human Reproductive Aging
}

\section{Citation}

Ellison, Peter T. 2010. Life historical perspectives on human reproductive aging. Annals of the New York Academy of Sciences 1204: 11-20.

\section{Published Version}

doi:10.1111/j.1749-6632.2010.05611.x

\section{Permanent link}

http://nrs.harvard.edu/urn-3:HUL.InstRepos:4703923

\section{Terms of Use}

This article was downloaded from Harvard University's DASH repository, and is made available under the terms and conditions applicable to Open Access Policy Articles, as set forth at http:// nrs.harvard.edu/urn-3:HUL.InstRepos:dash.current.terms-of-use\#OAP

\section{Share Your Story}

The Harvard community has made this article openly available.

Please share how this access benefits you. Submit a story.

\section{Accessibility}




\section{Commentary: Life historical perspectives on human reproductive aging}

Peter T. Ellison

Department of Human Evolutionary Biology

Harvard University

All of the papers in this section are framed in terms of life history theory, the branch of evolutionary biology that seeks to understand the evolution of mortality and fertility patterns. The phenomenon of reproductive aging clearly falls within this domain, but the facts of human reproductive aging have been difficult to square with the dominant life history models. A long post-reproductive life, in particular, has been viewed as an anomaly in need of explanation. The papers in this section range from theoretical treatments of general and reproductive senescence to more specific attempts to grapple with the evolution of human reproductive aging in particular. In this commentary I will make a few observations on some of the key contributions of these papers, and then offer a few related observations of my own.

Kirkwood and Shanely [1] provide an excellent overview of the evolutionary theory of senescence. One of their principal points is to underscore that senescence decline in somatic function - is a consequence of the inevitable accumulation of mortality, rather than mortality being a result of the inevitable accumulation of senescence. Because of diminishing survivorship the fitness value of late-age investment in somatic maintenance and repair necessarily declines. Organisms that devote energy and other limiting resources to preserving somatic integrity for an old age that rarely comes are clearly at a selective disadvantage.

Kirkwood and Shanely suggest that reproductive aging should be viewed as a variant of senescence, a decline in the functional capacity of the germ cells, their supporting tissues, and/or other somatic components of the reproductive system. If reproductive tissues themselves require maintenance and repair, including the germ line itself, then the general evolutionary theory of senescence would apply. Once again the expectation, almost universally met, is that organisms in the wild will ordinarily die before reproductive senescence causes an extreme deterioration of fecundity. Maintaining fecundity for an old age that rarely comes is also energetically wasteful, and therefore deleterious for fitness.

The problem, then, is to understand the few cases in which the expectation is not met, particularly humans, where a long post-reproductive life is common. Kirkwood and Shanely make two observations that should be incorporated by any evolutionary theory of post-reproductive life:

(1) There is little or no value to post-reproductive life unless it is not truly "postreproductive" but rather includes a capacity for continued reproductive investment.

(2) There is little or no cost to post-reproductive life unless post-reproductives are somehow "parasitic," lowering the fitness of their offspring and relatives. 
They point out that while there is considerable evidence for continued reproductive investment by post-reproductive individuals, it is unlikely to have greater fitness consequences than continued direct reproduction would have had, and therefore cannot provide a "causal" explanation for the emergence of post-reproductive life.

Tuljapurkar and Steiner [2] take on the question of phenotypic heterogeneity in fitness traits among individuals. Most life history models, like most demographic and population genetics models, incorporate assumptions of "fixed heterogeneity," e.g., a fixed distribution of fecundity among couples or of frailty among individuals.

Tuljapurkar and Steiner note that, in fact, the phenotypes of individuals change over time in trajectories that incorporate large stochastic components. They turn their attention to the development of alternative models that take such "dynamic heterogeneity" into account.

Dynamic heterogeneity models must surely represent a promising direction, very much in keeping with contemporary efforts to better understand phenotypic variation as something over and above genetic variation. The model developed so far by Tuljapurkar and Steiner might be termed a "fully stochastic" model in which fitness traits of interest are allowed to vary stochastically and independently at the level of the individual. They find that data from a population of English swans fits a dynamic heterogeneity model better than a fixed heterogeneity model. That, of course, does not mean that their model is the best possible. I hope and expect that, as this family of models matures, Tuljapurkar and Steiner and others will investigate other possible structures for dynamic heterogeneity. One important variant would be a model in which positive correlations between fitness traits are possible. This would reflect the phenomenon of "phenotypic correlation" in which traits that one might expect to be negatively correlated -- survival and fertility, for example -- are in fact positively correlated. This often occurs, for example, when both traits are affected by common conditions of energy abundance or scarcity. Other sources of phenotypic variation, including epigenetic developmental plasticity, might also be incorporated into future versions of the dynamic heterogeneity models.

Bribiescas [3] raises the issue of phenotypic plasticity in his paper on human male reproductive aging, noting that in some ways the reproductive system of the aging male appears to display a diminishing range of phenotypic responsiveness. Circadian variation appears to declines with age, for example, as does the anabolic response to exercise. Bribiescas also makes the important point, also noted in passing by Kirkwood and Shanely [1] and reiterated by Kaplan et al. [4] that human males do, in fact, display significant reproductive aging with minimal fertility in the sixth decade and beyond. Although male germ cells continue to be produced late in life, declining function of Sertoli cells, prostate and accessory glands, declining potency and libido all combine to dramatically curtail the fecundity of most males. Rather than thinking of males and females as being on very different trajectories of reproductive aging, their trajectories are in fact quite parallel, as predicted by Kirkwood's disposable soma theory [5] extended to reproductive tissues. The key sex difference is not in the fact of reproductive aging, but in the strategy of gamete production in the first place, contrasting a male strategy of 
persistent germ cell mitosis (which I term "iterogametogenesis" in parallel with the term iteroparous refering to repeated parity) with a female pattern of limited germ cell mitosis (which I correspondingly term "semelgametogenesis") resulting in a finite, and eventually depletable, oocyte supply. Virtually all mammals display this sex difference in gamete production, with the result that virtually any female mammal, if kept alive long enough, will undergo true menopause.

Hawkes and Smith [6] underscore the fact that a limited oocyte supply together with a constant rate of atresia will necessarily result in menopause in any long-lived female mammal. They go further by reviewing evidence to indicate that the rate of atresia in chimpanzees is virtually identical to that in humans and the predicted average age of oocyte depletion virtually the same. Emery Thompson et al. [7] report that late-age fecundity and late-age survivorship may be correlated in chimpanzees, based on a relatively small sample of wild animals, and from this observation speculate that reproductive aging may be quantitatively different in chimpanzees compared to humans. But Hawkes and Smith point to similar observations among Utah Mormons. Indeed, as noted above, the observation of phenotypic correlation between survivorship and fertility at the individual level should not surprise us, whether in chimps or humans. Hawkes and Smith in contrast argue convincingly that the fixed oocyte supply and rate of atresia observed in humans should be considered and ancestral trait, not a derived one. Rather the extended adult life expectancy of humans is the derived trait. If anything about human menopause needs an evolutionary explanation it is not its appearance, but rather it's persistence under conditions of a dramatically extended adult life expectancy.

Kaplan, Guerven, Winking, and Hooper [4] directly address the issue of postreproductive life and the persistence of menopause in humans, approaching it from the perspective of a life-course energetic model wherein the energetic costs of fertility must be supported by caloric productivity in excess of consumption at the family level. Kaplan and his various colleagues have been developing models of this type for some time with considerable success in both empirical and theoretical terms [8]. They use demographic and behavioral data from a number of well-studied forager populations to inform their models. One general observation supported by these models is that young individuals consume more than they produce while older individuals produce the surplus necessary to support the young. This surplus production is enough to maintain a relatively high rate of fertility for humans. Here they use these models to argue that, compared to the current reality of female menopause around age fifty, any extension of female fecundity into the sixth decade would increase total caloric demand by increasing the number of offspring dependent on old parents while diminishing productivity to the point of pushing families into net caloric deficit, an unsupportable scenario. They also note that male productivity is sustained at much higher levels than female productivity late in life even as male fertility declines to insignificance. The interpretation they advance is that the diversion of energy flows from late age individuals to current offspring and grand-offspring is favored by natural selection whereas the extension of female fecundity is not. They also argue that the diversion of male productivity in this 
way is quantitatively much more important that the diversion of female productivity, though the principle holds for both sexes.

In terms of Kirkwood and Shanley's [1] two observations, Kaplan et al. find positive benefit in the continued reproductive investment of post-reproductive individuals, but primarily in post-reproductive males rather than females. Thus they would attribute positive selective pressure for post-reproductive life primarily to a "grandfather effect." They also find negative fitness consequences of extended female fecundity, both in terms of increased numbers of dependent children and decreases in late-age female productivity.

\section{Phenotypic variation in patterns of reproductive aging}

The papers in this section make a very coherent set with a number of overlapping themes and specific arguments that dovetail nicely. I would like to make a few additional points that I hope complement those already made.

My first observation pertains to the notion of phenotypic variation in reproductive aging. One of the most important contributions of reproductive ecology, and physiological ecology in general, to our understanding of human biology has been to emphasize the broad range of observable phenotypic variation associated with different environmental and developmental conditions. Perhaps the best example of this is variation in female ovarian function associated with variation in energy availability. Far from being a homeostatically canalized trait, human ovarian function, characterized by hormonal profiles across the menstrual cycle, is facultatively variable within individuals. A continuum of ovarian function has been described ranging from an idealized, "textbook" menstrual cycle through stages described as follicular suppression, luteal suppression, ovulatory failure, oligomenorrhea, and amenorrhea [9] (Figure 1) This continuum can be rendered quantitatively by measures of ovarian steroid hormones, particularly estradiol and progesterone, made across the entire cycle. Variation along the continuum represents variation in fecundity, or the probability of successful conception given exposure to intercourse, a probability that drops to zero if ovulation doesn't occur, but which is also quantitatively lower when hormonal profiles are suppressed [10-12].

Individual variation along the continuum of ovarian function is highly correlated with variation in metabolic energy availability. Even moderate decreases in energy balance or increases in energy expenditure are regularly observed to result in decreases in ovarian function [13-15]. This variation has been documented under a wide variety of conditions in a wide variety of populations [16-20]. Within any given population individual women probably form a distribution along the continuum of ovarian function and the distribution as a whole may shift in response to prevailing conditions that affect most people (Figure 2). For example, seasonal changes in energy balance associated with seasonal changes in food availability in subsistence populations have been linked to seasonal variation in the frequency of ovulation and seasonality in conceptions and births $[21,22]$. Developmental effects have also been documented [23]. Smaller size at birth has been association with lower levels of ovarian function in adulthood in Polish women 
[24, 25]. Bangladeshi migrants to London have higher levels of ovarian function as adults if they migrate as children compared to those who migrate as adolescents or adults [26].

As Bribiescas notes [3], variation in male testicular function appears to be less acute in response to short-term, moderate variations in energy availability. Variation between populations in average testosterone levels, however, does appear to reflect shifting distributions of male testicular activity in response to chronic energetic conditions [27, 28]. In both females and males variation in gonadal function associated with energetics can be interpreted as facultative modulation of reproductive effort [2931]. In females, variation in ovarian function modulates the probability of conception and thus the rate of reproduction and its high metabolic demands. In males, variation in testicular function modulates somatic investment in sexually dimorphic muscle mass as well as behavioral investment in mating effort.

In addition to variation correlated with energetics, both sexes show consistent patterns of variation in gonadal function associated with age [27, 32-34]. In females, ovarian function follows a parabolic trajectory with age, peaking in the early to mid twenties and declining noticeably by the mid to late thirties. In males, testosterone levels peak in the early twenties and decline steadily from there.

Importantly, however, the trajectory of decline in gonadal function in both sexes depends on the height of the peak attained in early adulthood. By the sixth decade ovarian function ceases in women of all populations, and testosterone levels in men of all populations become similarly low. But populations differ in the average rate of decline. In males, this appears to have significant consequences for changes in body composition [35]. In populations where testosterone levels decline substantially with age the tendency toward increasing adiposity is greater. The consequences of differential rates of decline in ovarian steroids in women have not been explored but could be equally important. Population variation in the experience of menopausal symptoms, for example, might well be influenced by the quantitative nature of changes in circulating ovarian steroids. Women in westernized, developed societies may experience these symptoms more acutely in part because of an experience of chronically higher exposure during their reproductive years.

It is also possible that changes in bone mineral metabolism will show variation associated with the rate of decline in circulating steroid levels. High exposure to ovarian steroids during the reproductive years may lead to lower receptor densities or other changes in tissue sensitivity, leaving individuals at greater risk of the consequences of steroid withdrawal.

The take-home message is that although the biology of reproductive aging may be common to all human populations, the reality of reproductive aging may differ between individuals and populations depending on the ecological conditions under which they live. Phenotypic heterogeneity in gonadal function is associated with ecological conditions for adaptive reasons, and because environments differ, so will patterns of reproductive aging. 


\section{Energy flows and human demography}

Most contemporary models of human evolution incorporate the observation that human reproductive rates are high and birth intervals short compared to other hominids. The energetic burden of this reproductive rate is higher than an individual female can sustain based on her own metabolic productivity. Thus most evolutionary models consider energetic inputs in addition to the mother's to be crucial to her reproductive success and the emergence of modern human life history patterns. Different scholars have championed different candidates for the source of this additional energetic input. Lovejoy [36] hypothesized the importance of contributions from monogamously pairbonded males. Hawkes and colleagues [37-39] have emphasized contributions from maternal grandmothers. Hrdy [40, 41] has included contributions from female peers, especially female relatives. Kramer [42] has emphasized the contributions of children in underwriting their own costs in the family. In their chapter of this volume, Kaplan et al. $[4,43]$ emphasize the contributions of males, highlighting those of post-reproductive males in particular. Hill and Hurtado [43] argue that energetic contributions from outside a mated pair are nneccessary to support human reproductive rates while also arguing that grandmothers cannot quantitatively account for all of this input. Reiches et al. [44] propose that humans are characterized by a broad network of shared productive activities that essentially result in a pooled energy budget that all members of a cooperating productive/reproductive unit share in. At different stages of life all individuals may contribute energy surpluses to this pooled budget, either through their own efforts or through liberating more productive members of the group from less productive tasks. Indirect reproductive effort thus becomes an energy allocation category available to juveniles and adults as well as post-reproductive individuals, with consequences that resolve many of the anomalies of human life history patterns.

Discriminating among these competing hypotheses is not easy. Demographic models and assumptions are often invoked and challenged by those engaged in debate over these issues. For example, Hill and Hurtado [45] argue that the probability of living, co-resident grandmothers is too low for their contributions to have a major effect, while Hrdy $[40,41]$ argues against assumptions of patrilocality among proto-humans. The highly specific models of Kaplan et al. [4] and Hill and Hurtado [43]use observed fertility and mortality rates among specific extant foragers to model energy consumption and production across the lifespan. The gains in precision from this style of modeling have costs in making the resulting models less generalizable (Levins 19XX).

An additional demographic argument can be made, relevant to this debate, that does not depend on any specific assumptions about levels of mortality or fertility or patterns of residence, based simply on the probabilities of living relatives of various kinds and the way those probabilities change with changing conditions of mortality and fertility. The formal mathematics of these probabilities has been developed by Keyfitz [46], but the key points are intuitive. (See also Howell's [47] use of these probabilities in analyzing kinship networks amog the Dobe !Kung.) One can think of relatives in three categories: ascendant lineal relatives (parents, grandparents, etc.), descendant lineal 
relatives (offspring, grandoffspring, etc.), and collateral relatives (uncles, aunts, brothers, sisters, cousins, nephews, nieces, etc.). One can then ask how, for a proband of a given age, the probability of having a living relative of a given type varies with changes in mortality rates and fertility rates.

The most important difference is this regard is between ascendant relatives on the one hand and collateral and descendant relatives on the other. The probability of living ascendant relatives depends only on mortality rates and is independent of fertility rates. Any individual has at most two living parents, four living grandparents, etc., and the expected number alive depends only on adult mortality rates. The number of current mates is usually limited in the same way (given the predominant pattern of serial monogamy among humans). The numbers of collateral and descendant relatives are not limited, however, and are sensitive to both fertility and mortality rates, particularly preadult mortality rates.

This simple demographic reality has consequences for any model of supplemental energetic inputs to human reproductive success. As fertility rates increase and mortality rates (particularly pre-adult mortality rates) decrease from chimpanzee-like levels to modern human levels, the numbers of expected collateral and descendant relatives increase markedly. The number of expected living ascendant relatives may increase as well, but can never exceed two parents, four grandparents, etc. On the other hand, the number of descendant relatives who are potential recipients of parental and grandparental inputs increases rapidly, diluting the potential contributions from ascendant relatives to any given proband. One can conclude, then, that as fertility levels increased in the human lineage, energetic contributions from collateral and descendant relatives likely became increasingly important for reproductive success and the contributions of ascendant relatives of diminishing importance.

\section{Climbing Mount Postreproductive Life}

I believe Hawkes and Smith [6] are correct in arguing that the ovarian physiology of female reproductive aging is an ancestral, and not a derived, trait in humans. It is not the timing of female reproductive aging that has changed since the last common ancestor of chimpanzees and humans (the "LCA"), but rather the rate of adult mortality. The evolutionary cause of our extended lifespan is another puzzle that would lead beyond the focus of this chapter, but it is likely that it did not occur until millions of years after the split from the chimpanzee lineage. It is useful, however, to contemplate the situation that arose when lifespan first began to extend beyond the end of reproduction. What selection pressures can we infer as a consequence when significant numbers of post-reproductive individuals begin to appear?

I can think of three possibilities:

1. To the extent that post-reproductive individuals are parasitic on younger relatives, decreasing their fitness, there would be selection to increase postreproductive mortality to remove them. 
2. There would be selection to extend reproductive life.

3. There would be selection on post-reproductive individuals to make a net positive contribution to the fitness of their younger relatives, over an above any costs that their presence imposes.

Often we are tempted, when confronted with alternatives like these to try to calculate the relative fitnesses of each option in some absolute fashion, like comparing the heights of different adaptive peaks on a Sewall Wright-style fitness landscape. That is, however, the wrong way to approach the question, as Wright himself argued. Natural selection does not act to move a population toward the highest peak, it moves it in the direction of the steepest immediate path of ascent, a path that may well lead to a peak that is not the highest in the neighborhood. So in this case we should not ask ourselves, "Which is highest, Mount Parasitic Elimination, Mount Extended Fecundity, or Mount Indirect Reproductive Effort?" Rather we should ask which provides the steepest initial path of ascent.

The slope of the path up Mount Parasitic Elimination depends on how serious the parasitism is, and can only result in restoring fitness to what it was before the parasitic elders appeared. The path up Mount Extended Fecundity may be very shallow initially, or may even lead through an impassable valley. For females, it would involve changing either the initial oocyte supply or the rate of follicular atresia. Because the rate of atresia is exponential, increasing follicular supply is very ineffective in producing changes in the ultimate age at follicular exhaustion. Doubling the follicular supply, the equivalent of two additional ovaries, would only delay the age of menopause by three or four years at most. Changing the rate of atresia would be more effective, but this trait seems to be highly conserved.

The path up Mount Indirect Reproductive Effort seems likely to be the steepest of the three, assuming that by the time extended lifespan appears humans are already living in groups with pooled energy budgets represented by cooperative foraging and food sharing. Selection would favor those post-reproductive elders who engaged in indirect reproductive effort by making positive net contributions to their relatives' fitness. By this logic, extended lifespan creates the selective pressure for grandparental contributions to their offspring, not the other way around, as is often argued.

Two additional observations are worth making about this scenario. While Mount Extended Fecundity may not provide a viable path for females, it may be much more tenable for males given their very different gametogenic physiology. Some mixed strategy of direct and indirect reproductive effort may provide the steepest path for males. And the path up Mount Indirect Reproductive Effort may be made shallower by the presence of freeloaders, post-reproductive individuals who do not contribute much if anything to the pooled energy budget but whose inclusive fitness may benefit from the contributions of other post-reproductive individuals. 
In summary, armchair reasoning can only advance our understanding so far. In thinking about the evolution of patterns of human reproductive aging, however, it does help to assemble all the contributions of papers like the ones in this section and to consider their combined consequences. When we do, I think we come to appreciate the three observations made here: (1) phenotypic variation exists in patterns of reproductive aging that is expressed at the level of populations and that may be associated with chronic conditions of energy availability; (2) humans share in a pooled energy budget that helps to support an accelerated rate of reproduction and that increases the potential contributions of collateral and descendant relatives over ascendant relatives as it becomes more successful; and (3) the major change in human life history physiology since the LCA has been the extension of the adult lifespan, not any change in ovarian physiology, with the consequence that extended lifespan has produced selective pressure for the emergence of indirect reproductive effort among post-reproductive individuals, not the reverse.

\section{References Cited}

1. Kirkwood, T. B. L. \& D. P. Shanley. 2010. On the connections between general and reproductive senescence and the evolutionary basis of menopause and postreproductive life. Ann N Y Acad Sci.

2. Tuljapurkar, S. \& U. K. Steiner. 2010. Dynamic heterogeneity and life histories. Ann N Y Acad Sci.

3. Bribiescas, R. G. 2010. An evolutionary and life history perspective on human male reproductive senescence. Ann N Y Acad Sci.

4. Kaplan, H., et al. 2010. Learning, Menopause and the Human Adaptive Complex. Ann N Y Acad Sci.

5. $\quad$ Kirkwood, T. B. L. 1977. Evolution of ageing. Nature. 270: 301-304.

6. Hawkes, K. \& K. R. Smith. 2010. Do women stop early? Fertility decline in humans \& chimpanzees. Ann N Y Acad Sci.

7. Emery Thompson, M., et al. 2007. Aging and fertility in wild chimpanzees: implications for the evolution of menopause. Curr Biol. 17: 2150-2156.

8. Kaplan, H. S. \& A. J. Robson. 2002. The emergence of humans: the coevolution of intelligence and longevity with intergenerational transfers. Proc Natl Acad Sci U S A. 99: 10221-10226.

9. Ellison, P. T. 1990. Human ovarian function and reproductive ecology: New hypotheses. American Anthropologist. 92: 933-952.

10. Lipson, S. F. \& P. T. Ellison. 1996. Comparison of salivary steroid profiles in naturally occurring conception and non-conception cycles. Hum Reprod. 11: 2090-2096.

11. Venners, S. A., et al. 2006. Urinary estrogen and progesterone metabolite concentrations in menstrual cycles of fertile women with non-conception, early pregnancy loss or clinical pregnancy. Hum Reprod. 21: 2272-2280.

12. Baird, D. D., et al. 1997. Preimplantation hormonal differences between the conception and non-conception menstrual cycles of 32 normal women. Hum Reprod. 12: 2607-2613. 
13. Ellison, P. T. 1994. Salivary steroids and natural variation in human ovarian function. Ann N Y Acad Sci. 709: 287-298.

14. Ellison, P. T. 2008. Energetics, reproductive ecology, and human evolution. PaleoAnth. 2008: 172-200.

15. Jasienska, G. 2003. Energy metabolism and the evolution of reproductive suppression in the human female. Acta biotheoretica. 51: 1-18.

16. Jasienska, G. \& P. T. Ellison. 1998. Physical work causes suppression of ovarian function in women. Proc Biol Sci. 265: 1847-1851.

17. Ellison, P. T., et al. 1993. Population variation in ovarian function. Lancet. 342: 433-434.

18. Ellison, P. T., N. R. Peacock \& C. Lager. 1986. Salivary progesterone and luteal function in two low-fertility populations of Northeast Zaire. Hum Biol. 58: 473-483.

19. Panter-Brick, C., D. S. Lotstein \& P. T. Ellison. 1993. Seasonality of reproductive function and weight loss in rural Nepali women. Hum Reprod. 8: 684-690.

20. Vitzthum, V. J., et al. 2002. Salivary progesterone levels and rate of ovulation are significantly lower in poorer than in better-off urban-dwelling Bolivian women. Hum Reprod. 17: 1906-1913.

21. Bailey, R. C., et al. 1992. The ecology of birth seasonality among agriculturalists in central Africa. J Biosoc Sci. 24: 393-412.

22. Ellison, P. T., N. R. Peacock \& C. Lager. 1989. Ecology and ovarian function among Lese women of the Ituri Forest, Zaire. Am J Phys Anthropol. 78: 519-526.

23. Ellison, P. T. 1996. Age and developmental effects on human ovarian function. In Variability in human fertility. Rosetta, L. \& C. G. N. Mascie-Taylor, Eds.: 69-90. Cambridge University Press. Cambridge, UK.

24. Jasienska, G., I. Thune \& P. T. Ellison. 2006. Fatness at birth predicts adult susceptibility to ovarian suppression: an empirical test of the Predictive Adaptive Response hypothesis. Proc Natl Acad Sci U S A. 103: 12759-12762.

25. Jasienska, G., et al. 2006. High ponderal index at birth predicts high estradiol levels in adult women. Am J Hum Biol. 18: 133-140.

26. Nunez-de la Mora, A., et al. 2007. Childhood conditions influence adult progesterone levels. PLoS Med. 4: e167.

27. Ellison, P. T., et al. 2002. Population variation in age-related decline in male salivary testosterone. Hum Reprod. 17: 3251-3253.

28. Bribiescas, R. G. 1996. Salivary testosterone levels among Aché hunter/gatherer men and a functional interpretation of population variation in testosterone among adult males. Human Nature. 7: 163-188.

29. Ellison, P. T. 2001. On Fertile Ground. Harvard University. Cambridge, MA.

30. Ellison, P. T. 2003. Energetics and reproductive effort. Am J Hum Biol. 15: 342-

351.

31. Bribiescas, R. G. 2006. Men: Evolutionary and life history. Harvard University Press. Cambridge, MA.

32. Ellison, P. T. 1994. Advances in human reproductive ecology. Annu Rev Anthropol. 23: 255-275.

33. Ellison, P. T. 1996. Developmental influences on adult ovarian function. Am J Hum Biol. 8: 725-734. 
34. Lipson, S. F. \& P. T. Ellison. 1992. Normative study of age variation in salivary progesterone profiles. J Biosoc Sci. 24: 233-244.

35. Campbell, B. C., P. B. Gray \& P. T. Ellison. 2006. Age-related patterns of body composition and salivary testosterone among Ariaal men of Northern Kenya. Aging: clinical and experimental research. 18: 470-476.

36. Lovejoy, O. 1980. The origin of man. Science. 211: 341-350.

37. Hawkes, K. 2003. Grandmothers and the evolution of human longevity. Am J Hum Biol. 15: 380-400.

38. Hawkes, K. 2004. Human longevity: the grandmother effect. Nature. 428: 128129.

39. Hawkes, K., et al. 1998. Grandmothering, menopause, and the evolution of human life histories. Proc Natl Acad Sci U S A. 95: 1336-1339.

40. Hrdy, S. B. 2009. Mothers and others: The evolutionary origins of mutual understanding. Belknap/Harvard. Cambridge, MA.

41. Hrdy, S. B. 2001. Mother nature: A history of mothers, infants, and natural selection. Pantheon. New York, NY.

42. Kramer, K. L. 2005. Maya children: Helpers at the farm. Harvard. Cambridge, MA.

43. Hill, K. \& A. M. Hurtado. 2009. Cooperative breeding in South American huntergatherers. Proceedings of the Royal Society B.

44. Reiches, M. W., et al. 2009. Pooled energy budget and human life history. Am J Hum Biol. 21: 421-429.

45. Hill, K. \& A. M. Hurtado. 1996. Ache life history: The ecology and demography of a foraging people. Aldine Transaction. Hawthorne, NY.

46. Keyfitz, N. 1985. Applied mathematical demography. Springer. New York, NY.

47. Howell, N. 1979. Demography of the Dobe !Kung. Academic Press. New York. 


\section{List of Figures}

Figure 1: The continuum of ovarian function [9, 14]. Ovarian function, as characterized by ovarian steroid profiles across the menstrual cycle, varies in a progressive fashion through grades of follicular suppression, luteal suppression, ovulatory failure, oligomenorrhea (infrequent menstruation) and amenorrhea (absent menstruation). These grades represent different levels of fecundity. Individual women move across this trajectory with age, energetic status, and breastfeeding status. Ordinarily the clinical horizon for recognizing suppressed ovarian function is at the level of disrupted menstrual regularity, but fecundity may vary considerably before this clinical horizon is reached.

Figure 2: Population variation along the continuum of ovarian function [14]. The distribution of women along the continuum of ovarian function can vary between populations or within a population over time. Changes in the frequency of women at any given grade may indicate shifts in the overall population distribution that affect population level fecundity.

Figure 3: Seasonal variation in conceptions among the Lese of the Ituri Forest, Democratic Republic of the Congo [21]. Variation in conception frequency is associated with changes in energetic status among Lese women driven by seasonal changes in food availability. These changes in energetic status are in turn associated with shifts in the population distribution along the continuum of ovarian function. 